\title{
Combinación Xilacina, Tramadol, Diazepam y Ketamina como Protocolo Anestésico para Ovariohisterectomía Canina en Campañas de Esterilización y sus Efectos en las Constantes Vitales
}

\author{
Combination of Tramadol, Diazepam, Xylazine and Ketamine as Anesthetic \\ Protocol for Canine Ovariohysterectomy in Sterilization Campaigns \\ AND ITS EfFects on Vital SignS
}

\author{
Jhon David Huayta Huanca ${ }^{1,2}$, Alexander Ureta Escobedo ${ }^{1}$
}

\section{Resumien}

\begin{abstract}
El estudio tuvo como objetivo determinar los cambios en la frecuencia respiratoria y cardiaca, pulso, saturación de oxígeno, temperatura y presión arterial provocados por la combinación de xilacina, tramadol, diazepam y ketamina durante la ovariohisterectomía canina para su posible uso en campañas de esterilización. Diez perras entre 10 meses y 6 años de edad, de diferentes razas y tamaños, clínicamente sanas, con hemograma y leucograma normal y clasificados como ASA I y II, fueron sometidas a cirugía electiva de ovariohisterectomía canina con el mismo protocolo anestésico. Se recolectó información durante las seis etapas del proceso anestésico-quirúrgico. La frecuencia cardiaca y el pulso se mantuvieron dentro de los niveles normales. Tres pacientes presentaron taquipnea durante el mantenimiento. Se registraron episodios de hipoxia leve y la temperatura mostró un descenso gradual. Todos los pacientes presentaron hipertensión durante el mantenimiento y al término de la cirugía. No ocurrieron complicaciones en la recuperación anestésica. El protocolo anestésico tiene un bajo costo y, pese a los cuadros de hipertensión, puede emplearse para la realización de ovariohisterectomía canina en campañas de esterilización para pacientes ASA I y ASA II.
\end{abstract}

Palabras clave: tramadol, ovariohisterectomía, campaña, esterilización, anestesia, hipertensión

\footnotetext{
${ }^{1}$ Escuela Profesional de Medicina Veterinaria y Zootecnia, Facultad de Ciencias e Ingenierías Biológicas y Químicas, Universidad Católica de Santa María, Arequipa, Perú

${ }^{2}$ E-mail: david_16_90@hotmail.com
}

Recibido: 27 de abril de 2016

Aceptado para publicación: 29 de julio de 2016 
The study aimed to determine changes in respiratory and heart rate, pulse, saturation of oxygen, temperature and blood pressure caused by the combination of tramadol, diazepam, xylazine and ketamine during canine ovariohysterectomy for possible use in sterilization campaigns. Ten bitches between 10 months and 6 years of age of different breeds and sizes, clinically healthy, with hemogram and leucogram normal and classified as ASA I and II, were subjected to elective surgery ovariohysterectomy using the same anesthetic protocol. Physiological data was recorded during the six stages of the anesthetic-surgical procedure. Heart rate and pulse were within normal levels. Three patients presented tachypnea during maintenance. Episodes of mild hypoxia were observed. Body temperature showed a gradual decline. All patients presented hypertension during maintenance and the end of the surgery. There were no complications during the anesthetic recovery. The anesthetic protocol is inexpensive and despite cases of hypertension, it can be used to perform ovariohysterectomy in canine sterilization campaigns for patients ASA I and ASA II.

Key words: tramadol, ovariohysterectomy, campaign, sterilization, anesthesia, hypertension

\section{INTRODUCCIÓN}

El incremento de la población canina es una preocupación constante en la ciudad de Arequipa, Perú, por lo que la realización de campañas de esterilización es una de las medidas más apropiadas para su control; de allí la importancia de observar un protocolo anestésico que produzca un adecuado plano anestésico quirúrgico, una recuperación rápida, eficiente y de un costo económico bajo; sin que todo esto perjudique la salud del paciente.

Durante la anestesia, los procesos fisiológicos normales se ven afectados, pudiendo alterarse la homeostasis del animal(Rioja et al., 2013) y, por lo tanto, causar hipotensión, hipoxia e hipotermia, entre otros inconvenientes en el paciente anestesiado. Entre las causas que dan lugar a la hipotensión en el periodo perianestésico se encuentran el descenso de la contractilidad miocárdica, hipovolemia, vasodilatación, arritmias cardiacas y disminución del retorno venoso (Rioja et al., 2013). De la adecuada oxigenación de los tejidos dependerá el resto de funciones vitales. Las repercusiones clínicas están relacionadas con el daño celular que se produce al disminuir el aporte de oxígeno a las células, siendo más relevantes en cerebro, corazón, medula espinal, hígado o riñón (Rioja et al., 2013). La hipotermia es otra de las complicaciones anestésicas más frecuentes y tiene efectos negativos como la recuperación demorada, profundización del plano anestésico, reducción del nivel de consciencia, vasoconstricción periférica, arritmias y reducción de la contractilidad miocárdica (Burzaco, 2001; Rioja et al., 2013).

En la actualidad se cuenta con una amplia variedad de fármacos que se pueden adquirir y con su adecuada combinación se puede lograr una anestesia quirúrgica equilibrada. Tenemos opioides que aportan una excelente analgesia como el fentanilo, la morfina, el butorfanol y el tramadol, este último siendo un opioide sintético. Los dos primeros son productos con mucho control, por lo que su adquisición es limitada. El butorfanol es un opioide parcial que otorga seguridad, pero su elevado costo limita su uso para algunos pacientes; el tramadol no otorga una analgesia tan intensa en comparación con los anterio- 
Cuadro 1. Protocolo anestésico utilizado en el estudio

\begin{tabular}{lll}
\hline Premedicación & Inducción & $\begin{array}{l}\text { Mantenimiento (bolos de } \\
\text { carga) }\end{array}$ \\
\hline - Xilacina (Dormixyl, & - Diazepam (Diazepet, & - Diazepam (Diazepet, \\
Agrovet Market): 0.8 & Brouw er): $0.5 \mathrm{mg} / \mathrm{kg} \mathrm{IV}$ & Brouwer): $0.15 \mathrm{mg} / \mathrm{kg} \mathrm{IV}$ \\
$\mathrm{mg} / \mathrm{kg} \mathrm{IM}$ & - Ketamina (Ket-A-100, & - Ketamina (Ket-A-100, \\
- Tramadol (Tramadol, & Agrovet Market): $8 \mathrm{mg} / \mathrm{kg}$ & Agrovet Market): $3 \mathrm{mg} / \mathrm{kg}$ \\
Vitalis): $4 \mathrm{mg} / \mathrm{kg} \mathrm{IM}$ & IV & IV \\
& & - Xilacina (Dormi-xyl, \\
& & Agrovet Market): 0.3 \\
& $\mathrm{mg} / \mathrm{kg} / \mathrm{IV}$ \\
\hline
\end{tabular}

res, pero su uso en la premedi-cación junto con un tranquilizante crea una neuroleptoanalgesia muy útil, además su facilidad para adquirirlo y su costo lo convierte en una buena opción (Rioja et al., 2013). La xilacina es un tranquilizante agonista $\alpha-2$ adrenérgico de mucha difusión en medicina veterinaria; además, aporta analgesia visceral (Plumb, 2010), volviéndolo una opción más llamativa para procedimientos intracavitarios en comparación con otros tranquilizantes como las fenotiazinas. El diazepam (benzodiacepina) y la ketamina (anestésico disociativo) son fármacos cuyo uso se viene dando desde hace muchos años. Además, la literatura los detalla como fármacos con mucho margen de seguridad, por lo que sumado a su costo accesible y gran disponibilidad en el mercado, los convierte en buenas opciones para conformar un protocolo anestésico.

\section{Materiales y Métodos}

El estudio se realizó en noviembre y diciembre de 2015 en el Centro Quirúrgico de la Clínica Veterinaria de Pequeños Animales de la Universidad Católica de Santa María (UCSM), Arequipa, Perú. Los animales fueron proporcionados por el albergue «Huellitas en busca de amor»».
Se trabajó con 10 caninos hembras de diversas razas. Los animales tenían entre 10 meses y 6 años de edad, tamaño y peso variado, clínicamente sanos. En el análisis de laboratorio presentaron hemograma y leucograma normal, clasificados como ASA I y ASA II. Las perras fueron sometidas a cirugía electiva de ovariohisterectomía. El estudio fue aprobado por el Comité Institucional de Ética de la Investigación de la UCSM. El protocolo anestésico se indica en el Cuadro 1.

Para el monitoreo de la frecuencia cardiaca se colocaron electrodos de tres canales. Uno en el brazo derecho, otro en el brazo izquierdo y otro en el miembro posterior izquierdo. Se posicionó el pulsioxímetro en la lengua para los registros de saturación de oxígeno $\left(\mathrm{SpO}_{2}\right)$ y pulso. Para la evaluación de la temperatura se usó un termómetro por vía rectal. La evaluación de frecuencia respiratoria se realizó de forma visual y auscultatoria. La presión arterial fue medida colocando un manguito de presión en la arteria mediana a nivel de los carpos, en uno de los dos miembros anteriores.

La primera toma de registros de las funciones vitales se hizo antes de la premedicación (Etapa preoperatoria, PO), la segunda toma se realizó a los 15 min después 
de la premedicación (Etapa de premedicación, PM) y se procedió a la cateterización de la vena cefálica, aplicándosele ampicilina, $20 \mathrm{mg} / \mathrm{kg}$, por vía IV. La dosis de inducción se administró 25 min después de la premedicación y la tercera medición de constantes fisiológicas se hizo 3 min después (Etapa de inducción, IN). Los parámetros clínicos fueron evaluados cada 5 min durante la ovariohisterectomía (Etapa de mantenimiento, MA). Las funciones vitales fueron registradas nuevamente a los 5 min de concluida la cirugía (Etapa de término de la cirugía, TC) y cada 10 min durante la recuperación del plano anestésico (Etapa de recuperación anestésica, RA).

La frecuencia cardiaca, pulso, frecuencia respiratoria y temperatura se registraron en todas las etapas del procedimiento, pero el porcentaje de saturación de oxígeno y presión arterial solo durante las etapas de MA y TC. La saturación de oxígeno se registró en la etapa RA hasta que el paciente comenzó a realizar movimientos linguales.

Se calificó el tiempo de recuperación anestésico con la aparición de los reflejos interdigital, palpebral, y cuando el paciente realizaba movimientos tratando de incorporarse. Se aplicó estadística descriptiva de tendencia central (media) y dispersión (desviación estándar).

\section{Resultados}

Cuatro de los 10 pacientes no alcanzaron el plano anestésico quirúrgico adecuado, por lo que se les aplicó un bolo de carga (combinación ketamina-xilacina). El tiempo de recuperación promedio fue de 53.9 minutos. La media del tiempo operatorio fue de 49.7 minutos. La media de tiempo para la aplicación del primer bolo de carga fue de 25 minutos de iniciada la cirugía. El costo de los anestésicos para una perra de $10 \mathrm{k}$ fue de S/. 5.86 (aproximadamente US\$ 1.80).
Los valores de frecuencia respiratoria y cardiaca (x minuto) y los porcentajes de saturación de oxígeno se presentan en el Cuadro 2. Los valores de presión arterial sistólica (PAS), diastólica (PAD) y media (PAM) se presentan en el Cuadro 3. El pulso permaneció dentro de los rangos normales durante todo el procedimiento. La temperatura corporal sufrió un descenso gradual conforme transcurría el procedimiento quirúrgico, pero sin alteraciones relevantes.

\section{Discusión}

Todos los pacientes experimentaron un descenso en su frecuencia cardiaca basal después de ser premedicados (Cuadro 2). La literatura atribuye este cambio a la neuroleptoanalgesia creada por la xilacina y el tramadol (Sumano y Ocampo, 2006; Engel y García, 2008; Rioja et al., 2013). Nueve de los 10 pacientes mostraron un aumento en la frecuencia cardiaca luego de la inducción, efecto que también fue encontrado por Peña et al. (2007) y Flores et al. (2009), y que en este estudio se le atribuye a la ketamina (Laredo y Catalapiedra, 2001a; Engel y García, 2008; Plumb, 2010). La frecuencia cardiaca estuvo controlada durante las etapas de MA y TC, considerando la frecuencia cardiaca normal de 61-160 latidos por minuto (Belerenian et al., 2001; Engel y García, 2008; Rioja et al., 2013), debido a los efectos bradicardizantes de la xilacina, que se contraponen a los efectos taquicárdicos de la ketamina (Peña et al., 2007).

Siete pacientes presentaron taquipnea en la etapa $\mathrm{PO}$, y puede ser atribuido al estrés generado por las instalaciones de la clínica. La frecuencia respiratoria disminuyó en la etapa de PM en 9 de los 10 pacientes, ubicándose dentro de los rangos referenciales de 11-30 respiraciones por minuto (Engel y García, 2008). Durante la IN, uno presentó apnea, siendo asistido mediante ventilación controlada (8 ventilaciones/min) hasta la reaparición de ventilación espontánea, la cual 
Cuadro 2. Distribución de los valores hallados de frecuencia respiratoria y cardiaca y de $\mathrm{SpO}_{2}(\%)$ en los pacientes durante el procedimiento anestésico y quirúrgico

\begin{tabular}{lccccc}
\hline & Preoperatorio Premedicación & Inducción & Mantenimiento & $\begin{array}{c}\text { Término de la } \\
\text { cirugía }\end{array}$ \\
\hline $\begin{array}{l}\text { Frecuencia } \\
\text { cardiaca } \\
(\mathrm{x} \text { min })\end{array}$ & $100.2 \pm 14.6$ & $61.3 \pm 8.1$ & $99.7 \pm 28.5$ & $95.1 \pm 23.1$ & $80.3 \pm 18.8$ \\
$\begin{array}{l}\text { Frecuencia } \\
\text { respiratoria } \\
(\mathrm{x} \mathrm{min})\end{array}$ & $45.0 \pm 15.8$ & $23.0 \pm 8.2$ & $12.8 \pm 5.1$ & $25.9 \pm 7.9$ & $24.4 \pm 6.9$ \\
$\mathrm{SpO}_{2}$ & & & & & \\
$(\%)$ & & & $87.0 \pm 3.5$ & $93.1 \pm 4.7$ \\
\hline
\end{tabular}

Cuadro 3. Distribución de los valores hallados de presión arterial sistólica (PAS), diastólica (PAD) y media (PAM) en los pacientes durante el procedimiento anestésico y quirúrgico

\begin{tabular}{lccc}
\hline & PAS & PAD & PAM \\
\hline Mantenimiento & $179.6 \pm 10.9$ & $133.5 \pm 8.9$ & $147.9 \pm 8.0$ \\
Término de la cirugía & $170.9 \pm 11.6$ & $127.8 \pm 9.2$ & $142.4 \pm 10.1$ \\
\hline
\end{tabular}

se produjo a los 32 minutos (este paciente no fue excluido del grupo ya que el comportamiento de los demás parámetros fisiológicos fue bastante similar al de los otros pacientes). El apnea se pudo deber a la dosis alta de xilacina usada en la premedicación, sumado al efecto adverso de la ketamina que produce depresión respiratoria (Sumano y Ocampo, 2006; Plumb, 2010) o por una reacción adversa del paciente a alguno de los fármacos empleados (Engel y García, 2008). Durante la MA, tres pacientes presentaron un leve incremento en la frecuencia respiratoria, pudiendo atribuirse al dolor (Engel y García, 2008) generado por la tracción del ligamento suspensor del ovario. Ninguno de los pacientes presentó bradipnea durante la
MA ni TC, información también mencionada por Peña et al. (2007).

Ninguno de los pacientes presentó un registro inferior al $80 \%$ de saturación de oxígeno (Cuadro 2) durante la MA, resultado similar el encontrado por Peña et al. (2007) en los protocolos de su estudio. Los efectos en la $\mathrm{SpO}_{2}$ se pueden deber al mantenimiento con ketamina, la cual no solo carece de efectos depresores cardiopulmonares, sino que incrementa el volumen minuto, frecuencia cardiaca y presión arterial (Laredo y Catalapiedra, 2001a; Plumb, 2010), de allí que se puede inferir que se mejoraría la perfusión sanguínea de los tejidos del cuerpo sin alterar la captación de oxígeno por el sistema respiratorio. 
Los registros de temperatura durante la $\mathrm{PO}, \mathrm{PM}$ e IN se encontraron dentro de los rangos normales (Báez et al., 2007; Engel y García, 2008). En las etapas de MA y TC fueron disminuyendo, pero dentro de los límites esperados, ya que durante la anestesia es común que los pacientes pierdan entre 1 y $4{ }^{\circ} \mathrm{C}$ (Rioja et al., 2013). Esto se debe a que el metabolismo se encuentra reducido a un nivel basal y no debería existir actividad del músculo estriado; asimismo, debido a los efectos farmacológicos de la xilacina sobre los mecanismos termorreguladores (Sumano y Ocampo, 2006; Plumb, 2010; Rioja et al., 2013). Resultados similares también fueron encontrados por Flores et al. (2009) en uno de sus protocolos utilizando xilacina en la premedicación.

Según los límites de normotensión sugeridos por Burzaco (2001), Belerenian et al. (2001) y Thurmon et al. (2003), las mediciones de la PAS, PAD y PAM en el presente trabajo (Cuadro 3 ) fueron elevadas, posiblemente por el uso de ketamina, que aumenta la presión aórtica media, presión arterial pulmonar y presión venosa central, así como al efecto hipertensor inicial de la xilacina, aunque no se apreció el efecto hipotensor posterior de dicho fármaco (Laredo y Catalapiedra, 2001a,b; Sumano y Ocampo, 2006), quizás debido a la dosis empleada en el presente estudio. Si bien estos valores superan los rangos normales, ninguno de los pacientes presentó complicaciones. Resultados similares fueron encontrados por Peña et al. (2007) con el uso de ketamina. Thibaut et al. (2002) y Flores et al. (2009) también obtuvieron registros de hipertensión con la premedicación con xilacina, aunque utilizando propofol como agente inductor en el primer caso y tiletamina y zolazepan en el segundo caso.

No ocurrieron complicaciones durante la recuperación anestésica. Seis pacientes presentaron taquipnea, lo cual se atribuye a la fase de recuperación posoperatoria (Thurmon et al., 2003) y no por efectos de algesia. Según Sandoval et al. (2010), la ad- ministración preventiva de tramadol asociada a xilacina controla el dolor posquirúrgico en la ovariohisterectomía canina.

\section{Conclusiones}

- El protocolo propuesto permite obtener un plano anestésico adecuado.

- El protocolo produce cuadros hipertensores en los pacientes, pero ofrece seguridad en pacientes ASA I y ASA II.

- El protocolo anestésico tiene un costo económico relativamente bajo, por lo que puede ser considerado en campañas de esterilización masivas.

\section{Agradecimientos}

Se agradece al Mag. Guillermo Vásquez, al MVZ Luis Lam y a la UCSM por las facilidades brindadas en la realización de este trabajo. Asimismo, el apoyo brindado por los egresados Catalina Guzmán, Gianfranco Díaz, Anggy Gutiérrez y Rodrigo Melgar.

\section{Literatura Citada}

1. Báez P, Ruiz I, Restrepo L, Ruiz J. 2007. Comparación de dos protocolos anestésicos para ovariohisterectomía en perras sanas. Rev Colomb Cienc Pec 20: 425-430.

2. Belerenian G, Mucha C, Camacho A. 2001. Afecciones cardiovasculares en pequeños animales. Buenos Aires, Argentina: Inter-Médica. $360 \mathrm{p}$.

3. Burzaco O. 2001. Accidentes anestésicos y reanimación cardiopulmonar. Consulta Difus Vet 9(78): 71-78.

4. Engel J, García L. 2008. Manual del ATV (auxiliar técnico veterinario). Barcelona, España: Multimédica. $447 \mathrm{p}$.

5. Flores S, Zerpa H, Ascanio E, Rojas J, Briceño E, Arrieta D, Maniglia G 2009. Evaluación de la inducción anestésica con tiletamina/zolazepan en 
perros sometidos a diferentes protocolos de premedicación. Rev Fac Cienc Vet 50(1): 11-18.

6. Laredo F, Redondo J, Gómez R, Belda E, Cruz J. 2001a. La preanestesia; analgesia, inmovilización farmacológica, tranquilización y ansiolisis. Consulta Difus Vet 9(77): 37-50.

7. Laredo F, Cantalapiedra A. 2001 b. Técnicas de anestesia general inyectable TIVA. Consulta Difus Vet 9(77): 51-61.

8. Peña J, Sánchez R, Restrepo L, Ruiz J. 2007. Comparación de cuatro protocolos anestésicos para ovariohisterectomía canina en jornadas de esterilización masiva. Rev Col Cienc Pec 20: 260-268.

9. Plumb D. 2010. Manual de farmacología veterinaria. $6^{\mathrm{a}}$ ed. Buenos Aires: Inter-Médica. 1239 p.

10. Rioja E, Salazar V, Martínez M, Martínez F. 2013. Manual de aneste sia y analgesia de pequeños animales. España: Servet. $223 \mathrm{p}$.

11. Sandoval O, Herzberg D, Galecio S, Cardona L, Bustamante H. 2010.

Analgesia preventiva en hembras caninas sometidas a ovariohisterectomía: comparación del efecto analgésico de morfina y tramadol asociados a xilazina. Rev Cient (Maracaibo) 20: 138-143.

12. Sumano H, Ocampo L. 2006. Farmacología veterinaria. $3^{\mathrm{a}}$ ed. México: McGraw-Hill Interamericana. $1082 \mathrm{p}$.

13. Thibaut J, Rivera T, Ahumada F. 2002. Anestesia endovenosa en perros mediante el uso de propofol en dosis única, premedicado con acepromazinaatropina y xilazina-atropina. Arch Med Vet 34: 25-35.

14. Thurmon J, Tranquilli W, Benson G. 2003. Fundamentos de anestesia y analgesia en pequeños animales. Barcelona, España: Masson. 470 p. 